\title{
TORSION OF A CIRCULAR ANISOTROPIC BEAM WITH TWO LINEAR CRACKS WEAKENED WITH A CIRCULAR CAVITY
}

\author{
Etimad BayramoĞlu Eyvazov, Mecit ÖGe \\ Bartın University, Faculty of Engineering, Department of Mechanical Engineering, Bartın, Turkey \\ e-mail: mecitoge@bartin.edu.tr; mecitoge@yahoo.com
}

\begin{abstract}
Various components made of anisotropic materials (plast-mass, glass material, etc.) have been widely used in the production of modern mechanisms and machinery. Precise calculation of these elements, constituting the design, holds great importance. In general, fracture and distribution are essential issues in safety calculations. In this study, torsion of a beam with an $S$ oblast having outer and inner constraints as $L_{2}$ and $L_{1}$ circles with $R_{2}$ and $R_{1}$ radii, respectively, is investigated.
\end{abstract}

Keywords: anisotropic medium, orthotropic beams, isotropic beams, affine connections, conformal mapping functions

\section{Introduction}

There is no study available in the literature a solution of the mentioned in the abstract cases, since the contour $L_{1}$ is void of the mapping function. In this study, the solution of the problem is presented with numerical values.

Here, torsion of an area limited with an outer circle $L_{2}$ with radius $R_{2}$ and inner circle $L_{1}$ with radius $R_{1}$, having two linear cracks, is investigated. The coordinates of the end points of these cracks are taken as $\pm e$. Volumetric forces are neglected.

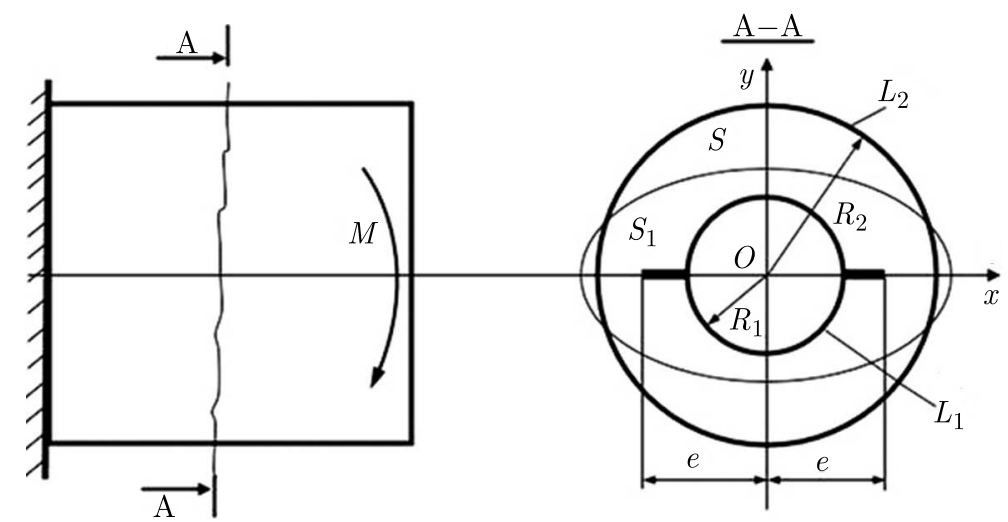

Fig. 1. Anisotropic beam and its cross-section after affine transformation

The beam is twisted by means of a torsional moment applied to the edges (Fig. 1). Here, the coordinate origin is taken as the center of cross-section. The beam is assumed to be made of a homogeneous anisotropic material. At least one elastic plane of symmetry is available on each point of the beam. In this case, all stresses except $\tau_{x z}$ and $\tau_{y z}$ are zero.

As known (Kosmodamianskii, 1976; Lethniskii, 1971; Kuliyev, 1991), solutions to torsion problems related to orthotropic beams are found using the solutions to torsion problems of beams with other cross-sections. In this case, affine connections are used 
$x_{1}=x \quad$ and $\quad y_{1}=\beta y$

It should be noted that if the affine connection is assumed as below, then the beam will not be orthotropic

$$
x_{1}=x+\alpha y \quad \text { and } \quad y_{1}=\beta y
$$

With expression (1.1), none of the horizontal values in the beam cross-section changes (since the horizontal axis does not change) as for the vertical values (i.e. those on $O y$ axis), they will either increase or decrease depending on the coefficient $\beta$ that characterizes anisotropy of the beam.

Thereby, in order to evaluate stresses on orthotropic bars, firstly, the torsion problem of an isotropic beam with $S_{1}$ cross-section (which is obtained with affine connection $=x_{1}$ and $y_{1}=\beta y$ ) should be solved.

According to the previous studies, $\tau_{x_{1} y_{1}}$ and $\tau_{y_{1} z_{1}}$ stresses are found from the following equation

$$
\tau_{x_{1} z_{1}}-\mathrm{i} \tau_{y_{1} z_{1}}=\mathrm{i}\left[2 F^{\prime}\left(z_{1}\right)-\bar{z}_{1}\right]
$$

where $\tau_{x_{1} z_{1}}$ and $\tau_{y_{1} z_{1}}$ are components of the tangential stresses on the cross-section $S_{1}$, and $\mathrm{i}$ - imaginary unit, $F\left(z_{1}\right)$ is the regular function on the cross-section $S_{1}, z_{1}=x_{1}+\mathrm{i} y_{1}$ and $\bar{z}_{1}=x_{1}-\mathrm{i} y_{1}$ are complex variables. $F\left(z_{1}\right)$ is calculated from boundary conditions, i.e., from the states of equilibrium and the equation of deformations on the boundaries.

These boundaries can be written as follows

$$
\begin{array}{lll}
\varepsilon_{x}=0 & \varepsilon_{y}=0 & \varepsilon_{z}=0 \\
\gamma_{x y}=0 & \gamma_{x z}=a_{55} \tau_{x z} & \gamma_{y z}=a_{44} \tau_{y z}
\end{array}
$$

where $a_{44}$ and $a_{55}$ are elastic constants that characterize anisotropy of the material.

Equations (1.1) with affine connections, semi axes of $L_{2}$ contour with radius $R_{2}$ are transformed into an ellipse $a=x_{1}$ and $b=\beta y_{1}$, on the other hand, the contour $L_{1}$ with radius $R_{1}$ on the $O x$ axis with two cracks, is transformed into an ellipse with two cracks (here it is assumed that $\beta \neq 1)$.

If $\beta<1$, then the linear values decline along the $O y$ axis, in the case of $\beta>1$ the same values increase.

$F\left(z_{1}\right)$ regular function within the enclosed area $S_{1}$ can be evaluated using the below given boundary conditions (Kosmodamianskii, 1976; Kuliyev, 1991, 2004; Sherman, 1992)

$$
F\left(z_{1}\right)-\overline{F\left(z_{1}\right)}=\mathrm{i} t_{1} \bar{t}_{1}+C_{k}
$$

where $t_{1}$ are affixes of the points on one of the contours of the cross-section $S_{1}$. $C_{k}$ is an arbitrary constant.

Components of $\tau_{x_{1} z_{1}}$ and $\tau_{y_{1} z_{1}}$ tangential stresses on characteristic points of the cross-section $S_{1}$ can be calculated by equation (1.4) (here, the end points of the cracks are also included).

Afterwards, $\tau_{x z}$ and $\tau_{y z}$ can be calculated for an orthotropic beam by the following equation (Kosmodamianskii, 1976; Lethniskii, 1971; Kuliyev, 1991)

$$
\tau_{x z}=\beta \tau_{x_{1} z_{1}} \quad \tau_{y z}=\tau_{y_{1} z_{1}}
$$

As indicated by these equations, $\tau_{y z}$ and $\tau_{y_{1} z_{1}}$ stresses do not vary on isotropic and anisotropic beams. Here, $\tau_{x z}$ tangential stress varies depending on the parameter $\beta$. It increases or decreases depending on $\beta\left(\beta=\sqrt{a_{44} / a_{55}}\right)$. 
$F\left(z_{1}\right)$ regular function can be expressed as follows for the contour $L_{2}$ within the enclosed $S_{1}$ area (Kosmodamianskii, 1976; Kuliyev, 1991, 2004; Sherman, 1992)

$$
F\left(t_{2}\right)=\mathrm{i} \sum_{k=0}^{\infty} \alpha_{k}\left(\frac{A_{2}}{t_{2}}\right)^{k}+\mathrm{i} \sum_{k=0}^{\infty} b_{k}\left(\frac{t_{2}}{A_{2}}\right)^{k} \quad \text { on } \quad L_{2}
$$

where

$$
\alpha_{k}=\sum_{\nu=k-2 E(k / 2)}^{k}{ }^{*} \alpha_{\nu} L_{(k-\nu) / 2} \quad b_{k}=\sum_{n=k}^{\infty} \beta_{k} a_{(n-k) / 2}^{(2)}
$$

$F\left(z_{1}\right)$ function can be defined as follows in the inner $L_{1}$ contour (an ellipse with two linear cracks) (Kosmodamianskii, 1976; Kuliyev, 1991; Sherman, 1992)

$$
F\left(t_{1}\right)=i \sum_{k=0}^{\infty} \alpha_{k} \xi_{1}^{-k}+\mathrm{i} \sum_{k=1}^{\infty} H_{1}(k) \xi_{1}^{k}+\mathrm{i} \sum_{k=0}^{\infty} H_{2}(k) \xi_{1}^{-k} \quad \text { on } \quad L_{1}
$$

The following notations are given in equation (1.7)

$$
\begin{array}{ll}
H_{1}(k)=\sum_{\nu=k}^{\infty} b_{\nu}\left(\frac{A_{1}}{A_{2}}\right)^{\nu} m_{1}^{\frac{\nu-k}{2}} C_{\nu}^{\frac{\nu-k}{2}} & H_{2}(k)=\sum_{\nu=\varepsilon}^{\infty} b_{\nu}\left(\frac{A_{1}}{A_{2}}\right)^{\nu} m_{1}^{\frac{\nu+k}{2}} C_{\nu}^{\frac{\nu+k}{2}} \\
\varepsilon^{\prime}=\varepsilon+\frac{1}{2}(k+\varepsilon) \quad \varepsilon=0 \quad \varepsilon=1 & \\
b_{k}=\sum_{n=k}^{\infty} \beta_{n} a_{(n-k) / 2} &
\end{array}
$$

The outer circle with contour $L_{2}$ (with semi axes $a_{2}=R_{2}$ and $b_{2}=\beta R_{2}$ ) is mapped on the circle with the unit diameter (equals to 1) using the mapping function (Kosmodamianskii, 1976; Kuliyev, 1991, 2004; Sherman, 1992)

$$
t_{2}=A_{2}\left(\tau+\frac{m_{2}}{\tau}\right) \quad A_{2}=\frac{R_{2}+\beta R_{2}}{2} \quad m_{2}=\frac{R_{2}-\beta R_{2}}{R_{2}+\beta R_{2}}
$$

The inner contour $L_{1}$ is mapped onto the circle with the unit diameter (equals to 1 ) using the mapping function (Kuliyev, 1991, 2004)

$$
t_{1}=A_{1} \tau \sum_{n=0}^{\infty} \tau^{-n} \Pi_{n}
$$

where

$$
\begin{array}{lrl}
A_{1}=\frac{R_{1}+\beta R_{1}}{2} & m_{1}=\frac{R_{1}-\beta R_{1}}{R_{1}+\beta R_{1}} \\
\Pi_{n}=\sum_{k=0}^{\infty} \gamma_{k-1} T_{n-k} & T_{n}=\sum_{\nu=n-2 E(n / 2)}^{n} m_{1}^{\frac{n-\nu}{2}} \gamma_{-1}^{\frac{n-\nu}{2}} l_{\nu}^{n-\nu}
\end{array}
$$

The inverse functions of (1.9) and (1.10) mapping functions are as bellow (Kosmodamianskii, 1976; Kuliyev, 2004; Sherman, 1992)

$$
\xi_{2}=\frac{z_{2}}{A_{2}} \sum_{n=0}^{\infty} a_{n}^{(2)}\left(\frac{A_{2}}{z_{2}}\right)^{2 n} \quad \xi_{1}=\chi(z)=\frac{z_{1}}{A_{1}} \sum_{n=0}^{\infty} E_{k}\left(\frac{A_{1}}{z_{1}}\right)^{k}
$$


The coefficients of the serial elements of the analytical functions $F_{1}(z)$ and $F_{2}(z)$ can be found using proper boundary conditions given below: (Kosmodamianskii, 1976; Kuliyev, 1991; Sherman, 1992)

$$
\begin{aligned}
& F_{1}\left(t_{1}\right)+\overline{F_{1}\left(t_{1}\right)}=\mathrm{i} \bar{t}_{1}+C_{1} \\
& F_{1}\left(t_{2}\right)+\overline{F_{1}\left(t_{2}\right)}=\mathrm{i} \bar{t}_{2}+C_{2}
\end{aligned}
$$

where $t_{1}$ and $t_{2}$ variables are properly found from (1.9) and (1.10) equations.

As we place equations (1.6) and (1.7) into boundary condition (1.12), we obtain a linear algebraic system depending on two unknown coefficients following some mathematical connections and remarks by Kosmodamianskii (1976), Kuliyev (1991, 2004). Here, we proceed with the variable $\tau$ since $\tau \bar{\tau}=1$ on the unit circle)

$$
\begin{array}{ll}
\alpha_{k}+H_{1}(k)+H_{2}(k)=\sum_{n=k}^{\infty} \Pi_{n-k} \Pi & \text { on } \quad L_{1} \\
V_{1}(k)+V_{2}(k)+V_{3}(k)=A_{2}^{2} m_{2} \varepsilon & \text { on } \quad L_{1}
\end{array}
$$

where $V_{1}(k), V_{2}(k), V_{3}(k)$ are respectively found from the following equations (Kuliyev, 1991, 2004)

$$
\begin{aligned}
V_{1}(k) & =\sum_{\nu=0}^{k}\left(\frac{A_{1}}{A_{2}}\right)^{k} C_{-\nu}^{\frac{k-\nu}{2}} m_{2}^{\frac{k-\nu}{2}} \alpha_{\nu} \quad V_{2}(k)=\sum_{\nu=k}^{\infty} b_{\nu} C_{\nu}^{\frac{k+\nu}{2}} m_{2}^{\frac{k+\nu}{2}} \\
V_{3}(k) & =\sum_{\nu=k}^{\infty} b_{\nu} C_{\nu}^{\frac{k-\nu}{2}} m_{2}^{\frac{k-\nu}{2}}
\end{aligned}
$$

From the first terms of these equations, a system of equations is obtained. $\alpha_{k}$ and $\beta_{k}$ are coefficients that can be found using these equations.

This is presented with the following numerical example.

Cross-sectional dimensions of the beam are assumed in accordance with the following ratio for numerical calculations.

1. In the case of $\beta=1 / 2$, the semi axes of the outer contour (curvilinear line, the circle with radius $R_{2}$ ) are transformed into the ellipse with semi axes $a_{2}=R_{2} ; b_{2}=\beta R_{2}$, and the inner contour (the one with two cracks and radius $R_{1}$ ) is transformed into the ellipse with two cracks. The semi axes of such ellipses can be defined as $a_{1}=R_{1} ; b_{1}=\beta R_{1}$. Accordingly, the problem related with torsion of the beam depending on the parameter $\beta$ is calculated using the torsion problem of another beam with a different cross section.

Tangential $\tau_{x z}$ and $\tau_{y z}$ stresses, given in Table 1, are found using equation (1.3).

Table 1. Tangential $\tau_{x z}$ and $\tau_{y z}$ stresses for choice 1

\begin{tabular}{|c|c|c|c|}
\hline Choice 1 & Points & $\tau_{x z} /\left(\mu \tau a_{2}\right)$ & $\tau_{y z} /\left(\mu \tau a_{2}\right)$ \\
\hline \hline$\beta=1 / 2$ & $z=0.65$ & - & 24.58 \\
$a_{1}=2 b_{1}$ & $z=0.70$ & - & 13.29 \\
$a_{1}=0.25 a_{2}$ & $z=0.75$ & - & 8.02 \\
& $z=1.00$ & - & 2.92 \\
\hline & $z=0.5 \mathrm{i} a_{1}$ & 0.548 & - \\
& $z=0.7 \mathrm{i} a_{1}$ & 0.141 & - \\
& $z=1.0 \mathrm{i} a_{1}$ & 0.118 & - \\
\hline
\end{tabular}


2. In the case of $\beta=1 / 2, R_{2}=0$. The inner contour transforms into the linear crack with length $l=2 e$; and the outer contour transforms into the ellipse with semi axes $a_{2}=R_{2}$, $b_{2}=\beta R_{2}$. This way, the problem, the subject of the current study, is solved by means of the solution to the torsion problem of the elliptical beam with a central linear crack.

The values of $\tau_{x z}$ and $\tau_{y z}$ tangential stresses for choice 2 are given in Table 2 .

Table 2. Values of $\tau_{x z}$ and $\tau_{y z}$ tangential stresses

\begin{tabular}{|c|c|c|c|}
\hline Choice 1 & Points & $\tau_{x z} /\left(\mu \tau a_{2}\right)$ & $\tau_{y z} /\left(\mu \tau a_{2}\right)$ \\
\hline \hline$\beta=1 / 2$ & $z=0.65$ & - & 14.17 \\
$b_{1}=0$ & $z=0.70$ & - & 7.76 \\
$a_{1}=0.5 a_{2}$ & $z=0.75$ & - & 4.77 \\
& $z=1.00$ & - & 1.56 \\
\hline & $z=0.5 \mathrm{i} a_{1}$ & -1.01 & - \\
& $z=0.7 \mathrm{i} a_{1}$ & -0.031 & - \\
& $z=1.0 \mathrm{i} a_{1}$ & -0.48 & - \\
\hline
\end{tabular}

\section{Conclusion}

Calculations of torsion of orthotropic beams can be performed using calculations of isotropic beams with different cross-sections (the cross-section $S$ is obtained with affine connection $x_{1}=x$ and $y_{1}=\beta y$ ). Here, the linear values on the $x$ axis do not vary with the varying parameter $\beta$ ), the ones on the $y$ axis increase or decrease in direct proportion with $\beta$.

The stresses on orthotropic beams can be calculated using the equations given below $\left(\tau_{x_{1} z_{1}}\right.$ and $\tau_{y_{1} z_{1}}$ are known)

$$
\tau_{x z}=\beta^{2} \tau \tau_{x_{1} y_{1}} \quad \tau_{y z}=\beta \tau \tau_{y_{1} z_{1}}
$$

\section{References}

1. Kosmodamianskit A.S., 1976, Stress State of Anisotropic Media with Holes and Cavities, Kiev-Donetsk Vysshaya Shkola, p. 200

2. Kuliyev S.A., 1991, Two Dimensional Problems of Elasticity Theory, M. Stroyizdat, p. 352

3. KuliYev S.A., 2004, Conformally Mapping Functions of Complex Domains, Baku Azerneshr, p. 372

4. Lethniskit S.G., 1971, Torsion of Anisotropic and Inhomogeneous Bars, M. Nauka, p. 200

5. Sherman D.I., 1992, Solution of planar elasticity problems in anisotropic media, Prikladnaya Mathematika i Mekhanika, 180-106 